\title{
Identification of prognostic genes in colorectal cancer through transcription profiling of multi-stage carcinogenesis
}

\author{
NING AN ${ }^{1 *}, \mathrm{CHEN}_{\mathrm{ZHAO}}{ }^{2 *}, \mathrm{ZHUANG}^{*} \mathrm{YU}^{1}$ and $\mathrm{XUE} \mathrm{YANG}{ }^{1}$ \\ ${ }^{1}$ Department of Oncology, Affiliated Hospital of Qingdao University, Qingdao, Shandong 266003; \\ ${ }^{2}$ Department of Anatomy, School of Basic Medicine, Qingdao University, Qingdao, Shandong 266071, P.R. China
}

Received November 14, 2017; Accepted July 9, 2018

DOI: $10.3892 / \mathrm{ol} .2018 .9632$

\begin{abstract}
Colorectal cancer is a complex multistage process following the adenoma-carcinoma sequence. Additional research on the basis of molecular dysregulations, particularly in the precancerous stage, may provide insight into the realization of potential biomarkers and therapeutic targets for the disease. In the present study, the expression profile of human multistage colorectal mucosa tissues, including healthy, adenoma and adenocarcinoma samples, was downloaded. Genes that were consistently differentially expressed in precancerous tissues and cancer samples were collected. Based on a merged biological network, the biggest connected component composed of these identified genes and their one-step neighbors were retrieved to conduct random walk with restart algorithm, in order to identify genes significantly affected during carcinogenesis. Therefore, 35 genes significantly affected by carcinogenic dysregulation were successfully identified. Survival and Cox analysis indicated that the expression of these genes was an independent prognostic factor confirmed by six cohorts. In summary, based on the transcription profile of multi-stage carcinogenesis and bioinformatics analysis, 35 genes significantly associated with patient survival were successfully identified, which may serve as promising therapeutic targets for the disease.
\end{abstract}

Correspondence to: Dr Xue Yang or Professor Zhuang Yu, Department of Oncology, Affiliated Hospital of Qingdao University, 16 Jiangsu Road, Shinan, Qingdao, Shandong 266003, P.R. China

E-mail: yxue0409@outlook.com

E-mail: yuzhuang2002@126.com

*Contributed equally

Abbreviations: CRC, colorectal cancer; DEGs, differentially expressed genes; RWR, random walk with restart; GEO, Gene Expression Omnibus; TCGA, The Cancer Genome Atlas; HPRD, Human Protein Reference Database; KEGG, Kyoto Encyclopedia of Genes and Genomes; OS, overall survival; FDR, false discovery rate; EM, eigengene of the module; GO, Gene Ontology

Key words: colorectal cancer, carcinogenesis, biological network, random walk, prognostic genes

\section{Introduction}

Despite advances in understanding the underlying molecular mechanism of colorectal cancer (CRC), CRC remains a leading cause of cancer-associated mortality worldwide (1). Patient survival has been reported to be excessively dependent on clinical stage at the time of diagnosis, and reduced sensitivity to chemotherapy remains a major challenge in the effective management of CRC (2). Therefore, the identification of novel molecules promoting CRC progression is required for the identification of promising prognostic biomarkers and potential therapeutic targets (3).

$\mathrm{CRC}$ is a complex bioprocess following the adenoma-carcinoma multistage sequence. Therefore, understanding the molecular dysregulations during the process of carcinogenesis, particularly during the precancerous stage, may assist in identifying potential prognostic biomarkers and candidate therapeutic targets. Differentially expressed genes (DEGs) consistently and continuously altered throughout the whole carcinogenic process may reduce potential noise created by large-scale heterogeneity in tumors.

Network-based analyses have been demonstrated to be substantially effective in identifying causal cancer driver genes $(4,5)$. Based on publicly accessible biological networks, a random walk with restart (RWR) algorithm was used in the present study to identify potential genes affected significantly by source genes. RWR is a network-based mathematical model simulating a random walker starting from seed nodes and advancing towards randomly selected neighbors at each step (6). Subsequently, once the network dynamics are stable, the probability of walking to each node in the network is also inclined to be stable. Therefore, novel candidate molecules receiving significant information flow were regarded to be significantly affected by source genes.

In the last decade, the rapid development of high throughput technologies has greatly promoted modern studies of molecular oncology (7). However, expression-profiling datasets are short of systematic consistency between different studies, probably due to different working platforms and lab protocols $(8,9)$. In order to evaluate the robustness of identified prognostic genes, four microarray datasets in the Gene Expression Omnibus (GEO) database and one dataset from The Cancer Genome Atlas (TCGA) database (https://tcga-data.nci.nih.gov/tcga/) were used as 
independent testing cohorts in the present study, increasing the accuracy and robustness of the analysis.

\section{Materials and methods}

Download of transcriptomic profile of colorectal carcinogenesis. The processed expression profile of 117 colorectal carcinogenesis samples and 52 samples with survival information was downloaded from GEO database with the accession number GSE71187 (microarray platform ID, GPL6480) (10). Adenocarcinoma is pathologically defined as submucosal invasion by tumors (11). The global expression profiles contained the expression values of 18,986 genes. All associated data were directly downloaded from the original publication (10). Furthermore, TCGA RNA sequencing data, GSE14333 (12), GSE17536 (13), GSE39582 (14) and GSE28722 (15) were also downloaded from their original publications in order to test the validity of the identified gene signature.

Identifying consistent DEGs at precancerous and cancer stages. An unpaired significance analysis of microarrays (SAM) algorithm (16) was used to identify the DEGs during the following two transitions, i.e., between precancerous progression of colorectal adenoma $(n=58)$ and normal mucosal tissues $(n=12)$, and between CRC $(n=47)$ and precancerous progression [false discovery rate $($ FDR $)<0.005]$. Genes that were simultaneously and continuously upregulated or downregulated at the aforementioned transitions were regarded as consistent DEGs and used for further analysis.

Establishing a merged a priori knowledge-based biological network. The gene-gene interaction information was obtained from combining the a priori knowledge from the Human Protein Reference Database (HPRD; http://www.hprd.org/) and Kyoto Encyclopedia of Genes and Genomes (KEGG; https://www.kegg.jp/). The gene regulatory network was subsequently established by merging HPRD and KEGG gene-gene interactions, including 10,340 nodes and 60,642 edges. All consistently dysregulated DEGs were projected onto this merged network and the largest connected component comprised of consistent DEGs and corresponding one-step neighbors was retrieved for further analysis.

RWR to identify genes significantly affected by carcinogenetic dysregulation. Consistent DEG $i$ was weighted with $z_{\mathrm{i}}$ as follows:

$$
Z_{i}=\phi^{-1}(1-P i)
$$

In this formula, $p_{\mathrm{i}}$ represented the $\mathrm{P}$-value of Cox regression analysis between the expression value of DEG $i$ and the patients' overall survival (OS) rate; $\Phi^{-1}$ represented the inverse standard normal cumulative distribution function $(17,18)$. Therefore, $z_{\mathrm{i}}$ increased monotonically along with OS association of DEG $i$, following a standard normal distribution. Gene-gene interactions were further weighted with corresponding co-expression values. For example, for a particular gene $x$ with the expression values $\left(x_{1}, \ldots \ldots, x_{n}\right)$ and another gene $y$ with the values $\left(y_{1}, \ldots \ldots\right.$, $\left.y_{\mathrm{n}}\right)$, the formula for the interaction weight $E_{\mathrm{r}}$ was as follows:

$$
E_{r}=\left|\frac{\sum_{i=1}^{n}\left(x_{i}-\bar{x}\right)\left(y_{i}-\bar{y}\right)}{\sqrt{\sum_{i=1}^{n}\left(x_{i}-\bar{x}\right)^{2}} * \sqrt{\sum_{i=1}^{n}\left(y_{i}-\bar{y}\right)^{2}}}\right|
$$

In RWR analysis, genes of interest were regarded as the information source, such as the source nodes, while the remaining genes were regarded as the target, such as the target nodes. Subsequently, the information flow was iteratively and randomly 'walked' from source nodes to their neighboring targets with a probability constantly proportional to their connectivity. Following considerable steps, the final steady-state probability calculated for each gene denoted the integrated influence imposed by source nodes, as well as network topology. The RWR algorithm was defined as follows:

$$
p^{t+1}=(1-r) W p^{t}+r p^{0}
$$

In this formula, $W$ is the column-normalized adjacency matrix of the target network and $p^{\mathrm{t}}$ is the probability vector the genes hold at step $t$ in the iterative process. Source nodes were initially weighted with the probability vector $p^{0}$, with the sum of all elements being 1 , and $r$ denoted the restart probability ( $r=0.7$ in the present study). All genes in the aforementioned network were ranked according to the values in the steady-state probability vector $p^{\infty}$. The iteration did not cease until the difference between $p^{\mathrm{t}}$ and $p^{\mathrm{t}+1}$ was $<1 \times 10^{-10}$, which was measured by the least absolute deviations norm. To increase the robustness of RWR analysis, 10,000 permutations of nodes were conducted to simulate the null distribution. Therefore, the P-value was designated as the ratio of random values greater than or equal to the observed final probability and then corrected using the FDR method. Genes with FDR of $<0.001$ were defined as the genes significantly affected by carcinogenic dysregulation.

Statistical analysis. Kaplan-Meier survival analysis was used to determine the OS difference between the two eigengene of the module (EM)-assigned groups. The first principal component (PC1) captured the greatest amount of total variance in the profiles and was calculated for each patient. Patients were then divided into two groups of equal size based on the rank order of PC1 across their tumor profiles. Kaplan-Meier survival analysis and the log-rank test were used to evaluate the prognostic difference between the two groups $(10,19)$. Cox regression model was conducted to evaluate the independence of prognostic factors. Samples along with their detailed information, including age, sex, stage and survival time, were used and $\mathrm{P}<0.05$ was considered to indicate a statistically significant difference. All data analyses were conducted using $\mathrm{R}$ programming (version 3.3.1; https://www.r-project. org/), Bioconducter packages (version 3.5; http://www. bioconductor.org/) and Matlab (version 2015b; https://www. mathworks.com/products/matlab.html) language. The network visualization and analysis were achieved through Cytoscape (version 3.4.0; http://www.cytoscape.org/). DEG identification was conducted with $\mathrm{R}$ package 'samr' (version 2.0; 

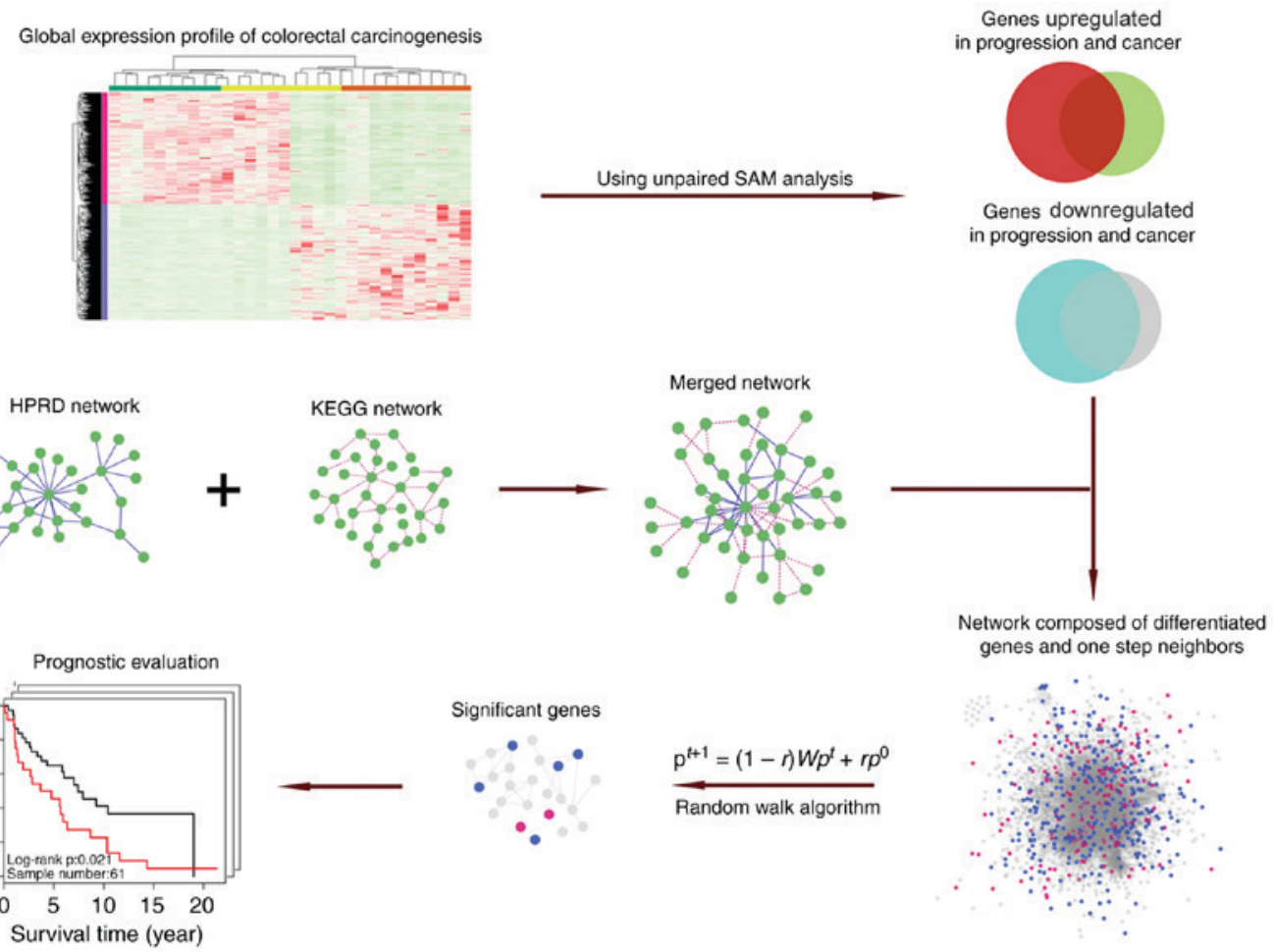

Figure 1. Schematic of methodology adopted in the present study. HPRD, Human Protein Reference Database; KEGG, Kyoto Encyclopedia of Genes and Genomes; SAM, significance analysis of microarrays.

https://cran.r-project.org/web/packages/samr/index.html), and meta-analysis was performed with R package 'meta' (version 4.8-2; https://cran.r-project.org/web/packages/meta/).

\section{Results}

Schematic of methodology. The schematic adopted for the present study is depicted in Fig. 1.

Consistently dysregulated DEGs in precancerous and cancer stages identified to reduce noise. The global expression profiles of human healthy colorectal mucosa, CRC precancerous (adenoma) and cancer (adenocarcinoma) samples from the GEO database (GSE71187) were downloaded. Using unpaired SAM analysis, 6,726 genes were upregulated and 4,825 genes were downregulated in precancerous samples compared with those in healthy tissues. A total of 2,404 genes were upregulated and 2,726 genes were downregulated in cancer samples compared with those in precancerous samples. Furthermore, DEGs that were simultaneously and continuously upregulated or downregulated during carcinogenic transitions were referred to as consistent DEGs. Therefore, 405 upregulated and 877 downregulated consistent DEGs were identified (Fig. 2A). Gene ontology (GO) analysis was further conducted with the Database for Annotation, Visualization and Integrated Discovery bioinformatics tool (http://david.abcc.ncifcrf.gov/). The results of the GO enrichment analysis indicated that the consistent downregulated DEGs were associated with the "cell development' process, while consistent upregulated DEGs functionally concentrated upon the 'immune response' and the 'defense response' in CRC (Fig. 2B).
Consistent DEGs are also dysregulated in the same direction in TCGA dataset. Gene set enrichment analysis using 32 paired RNA sequencing data of cancer and adjacent healthy tissue in TCGA database indicated that consistently upregulated DEGs were also significantly upregulated [normalized enrichment score $\left.(\mathrm{NES})=3.22 ; \mathrm{FDR}=2.20 \times 10^{-4} ; \mathrm{Fig} .3 \mathrm{~A}\right]$ and consistently downregulated DEGs were also significantly downregulated $\left(\mathrm{NES}=-3.36\right.$; FDR $=1.80 \times 10^{-4}$; Fig. $\left.3 \mathrm{~B}\right)$ in TCGA dataset Principal component analysis indicated that consistent DEGs retrieved from GSE71187 were able to distinguish CRC samples from adjacent healthy tissues in TCGA paired data (Fig. 3C and D).

Random walk in merged biological network to identify genes significantly affected by carcinogenic dysregulation. The identified consistent DEGs were projected onto the merged a priori knowledge-based biological network and subsequently, the largest connected component was obtained, composed of these consistent DEGs and their one-step neighbors (Fig. 4A). Finally, 35 genes significantly affected by carcinogenic dysregulation were successfully identified through the RWR algorithm (Fig. 4B). It was indicated that this network approximated the scale-free network topology of a transcriptional regulatory network (Fig. 4C), indicating that the merged network functioned as a small-world phenomenon (20). The largest connected component contained 173 consistently upregulated DEGs, 320 downregulated DEGs, 1,901 one-step neighbors and 21,691 interactions (Fig. 4D). Furthermore, DEGs and interactions were weighted with prognostic association by quantifying the survival association and with gene-to-gene co-expression by quantifying the biological affinity, using 52 CRC microarray data and corresponding 
A

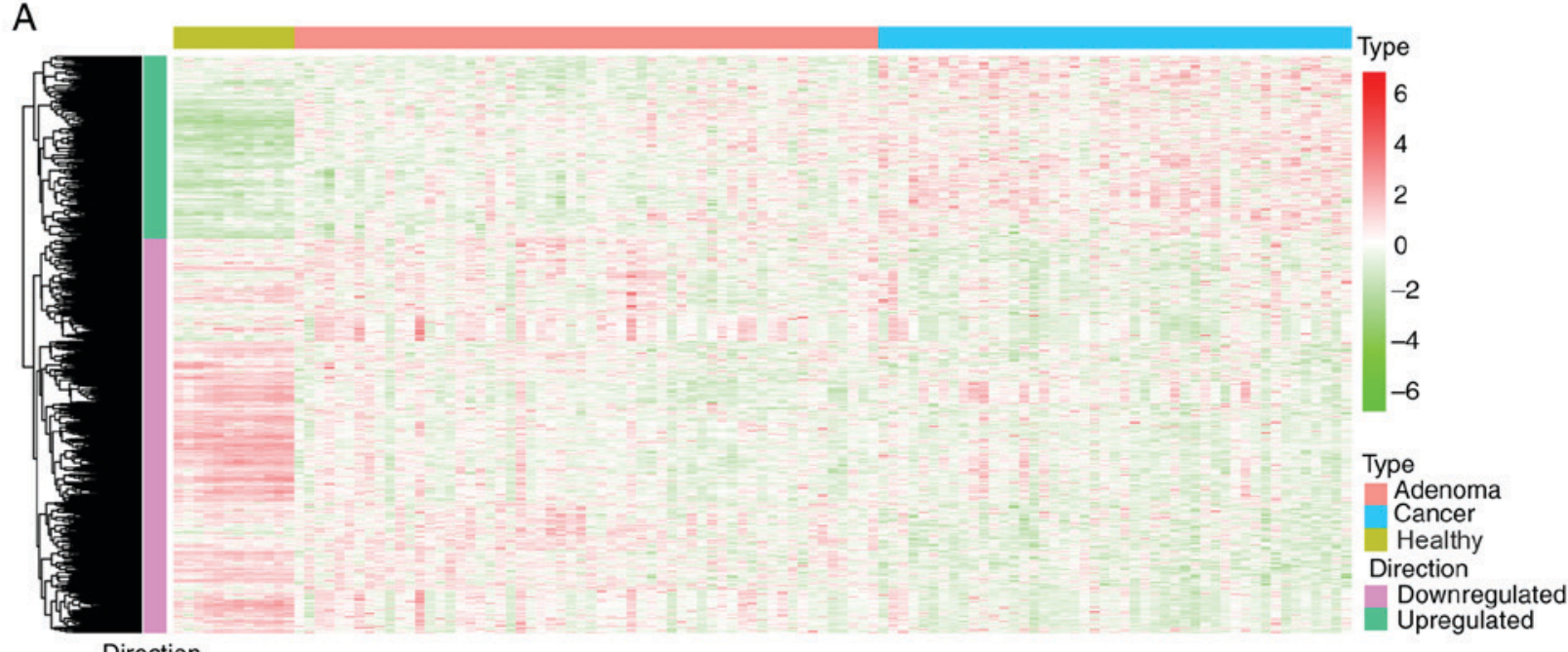

Direction

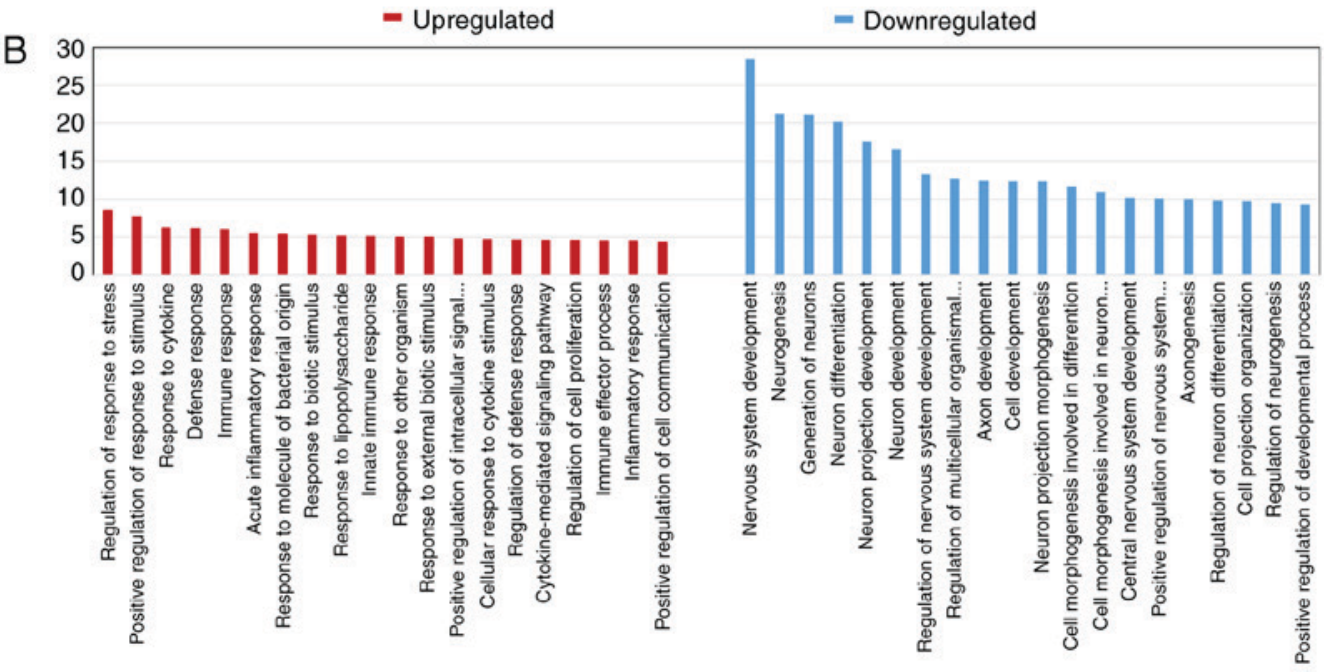

Figure 2. Collection of consistent DEGs and GO enrichment analysis. (A) Heatmap of 1,282 consistent DEGs, including 405 upregulated DEGs and 877 downregulated DEGs, in human healthy colorectal samples and in CRC precancerous and cancer samples. Unsupervised clustering algorithm was used to cluster DEGs with a similar expression pattern during colorectal carcinogenesis. (B) GO enrichment analysis of consistent DEGs. Bar length represented the-log10 transformed FDR value. The results revealed that upregulated consistent DEGs indicated the genes that were associated with 'immune response', while downregulated DEGs indicated the genes that were associated with 'cell development' processes. FDR, false discovery rate; DEGs, differentially expressed genes; CRC, colorectal cancer; GO, Gene Ontology.

survival information in GSE71187. The initial probability vector $p_{0}$ was calculated by normalizing score vector $(\mathrm{n}=493)$ so that the sum of the vector equaled 1 . When the steady state was achieved, genes in the connected component, including 493 source nodes, were scored with $p^{\infty}(\mathrm{n}=2,394$, output of random walk algorithm). Genes with significantly high score were referred to as the most affected ones by large-scale molecular dysregulations during carcinogenesis. Therefore, 35 genes significantly affected by carcinogenic dysregulation were collected through 10,000 permutations.

Validation of the prognostic value of the genes significantly affected by carcinogenic dysregulation. GSE71187 CRC survival data, TCGA RNA sequencing data, and datasets GSE14333, GSE17536, GSE39582 and GSE28722 were used to evaluate the prognostic value of the 35 genes significantly affected by carcinogenic dysregulation. The EM value of the candidate module was calculated based on their first principal component. Kaplan-Meier survival analysis was used to illustrate the difference of survival status between the two EM-assigned groups in each dataset. The result of the survival analysis indicated a satisfactory performance of the 35 genes significantly affected by carcinogenic dysregulation in all six independent cohorts (GSE71187, $\mathrm{n}=52$, $\mathrm{P}=0.028$; TCGA, $\mathrm{n}=377, \mathrm{P}=0.0068$; GSE14333, $\mathrm{n}=226, \mathrm{P}=0.016$; GSE17536, $n=177, P=0.0072$; GSE39582, $n=566, P=0.0073$; GSE28722, n=125, $\mathrm{P}=0.041$; Fig. 5). All genes were significantly dysregulated during CRC carcinogenesis in TCGA paired data $(\mathrm{P}<0.05$; Fig. 6$)$, with the exception of hepsin $(\mathrm{P}=0.350)$ and GLI family zinc finger $1(\mathrm{P}=0.906)$.

Confirmation of the prognostic value of the 35 genes significantly affected by carcinogenic dysregulation. Meta-analysis of 35 significant mRNAs in six independent cohorts was conducted with a fixed-effects model and a random-effects model (Fig. 7). The Cox proportional hazards regression model was used to evaluate the independence of the prognostic factors in a stepwise manner (Table I). In each independent cohort, samples with age, sex and American Joint Committee 
A

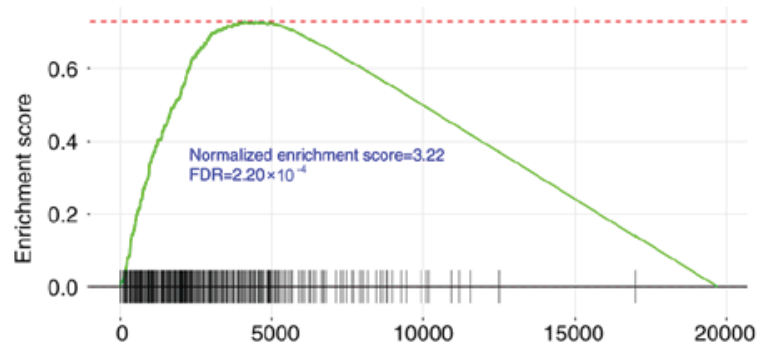

$\mathrm{C}$

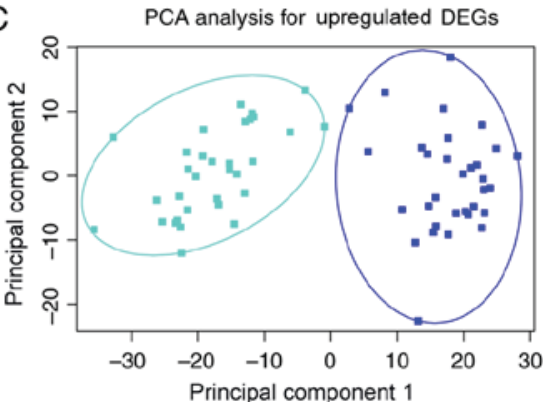

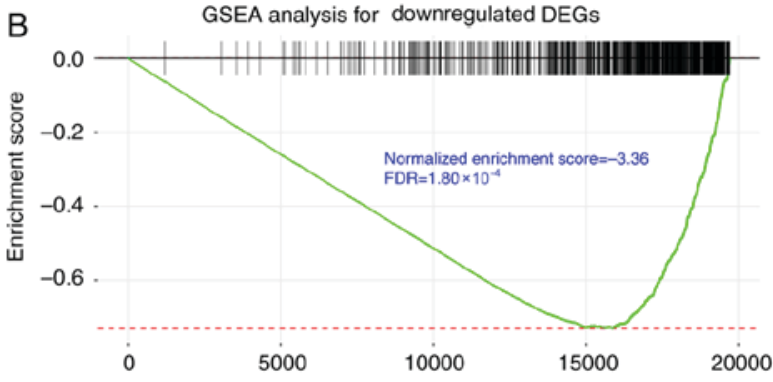

D

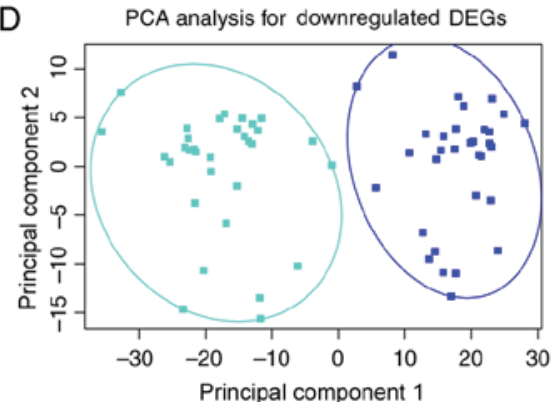

Figure 3. GSEA analysis of consistent DEGs using TCGA paired data set. GSEA analysis was conducted with the consistent (A) upregulated and (B) downregulated DEGs. The two gene groups revealed a concordant differential expression pattern in TCGA paired data sets (FDR $<0.001$ ). Principal component analysis was conducted with the consistent (C) upregulated and (D) downregulated DEGs, indicating that consistent DEGs retrieved in the GSE71187 dataset were able to distinguish CRC samples from adjacent healthy tissues. PCA, principal component analysis; GSEA, gene set enrichment analysis; FDR, false discovery rate; CRC, colorectal cancer; DEGs, differentially expressed genes; TCGA, The Cancer Genome Atlas.

B

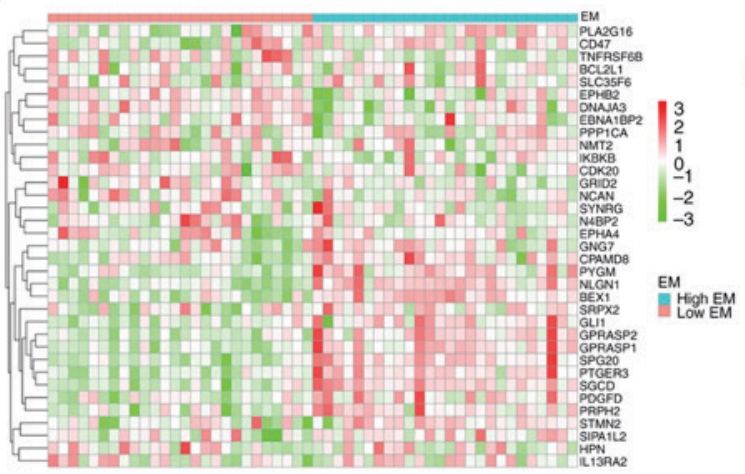

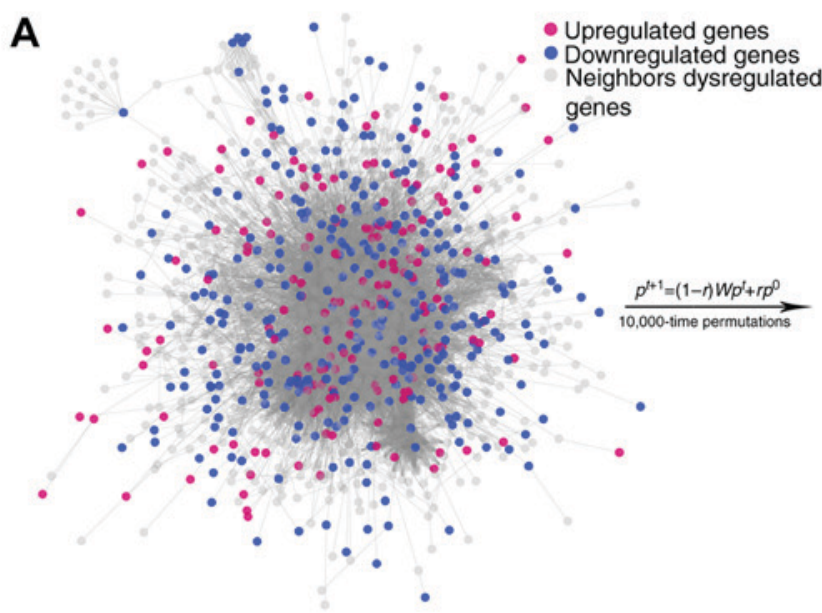

- Upregulated genes

Neighbors dysregulated

genes

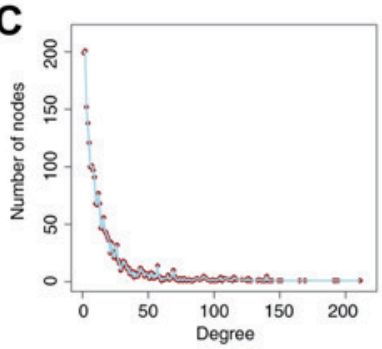

D

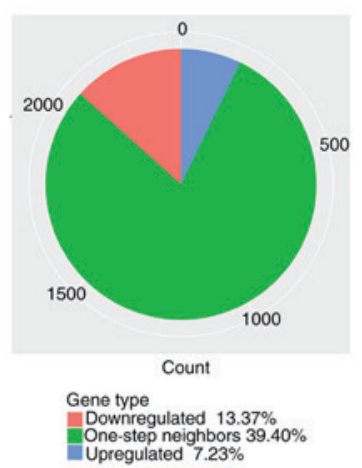

Figure 4. Random walk of consistent DEGs in merged biological network. (A) The biggest connected component containing 2,394 genes and 21,691 edges Consistent DEGs were regarded as source nodes and the rest genes in the network were target nodes. (B) The heatmap of the 35 genes in GSE71187. Rows represent 35 significant genes, which were clustered UCA, while columns represent samples, which are divided into two groups according to their corresponding EM value. (C) The network degree approximating the scale-free network topology of a transcriptional regulatory network. (D) Pie chart of 3 gene groups in the biggest connected component. UCA, unsupervised clustering algorithm; EM, eigengene of the module; DEGs, differentially expressed genes. 

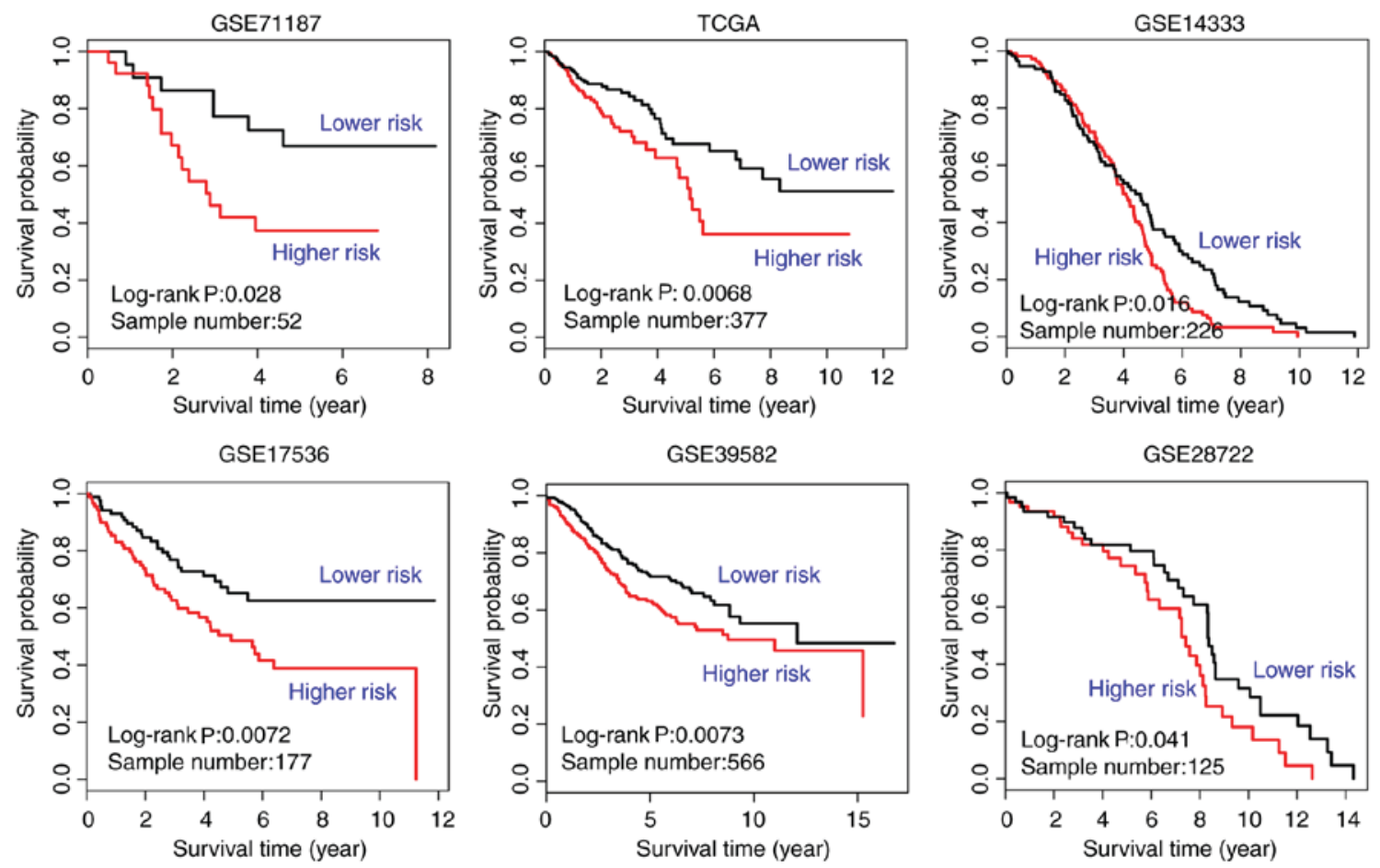

Figure 5. Kaplan-Meier survival analysis of the 35 significant genes in six study cohorts, of which the patients are divided into two EM-assigned groups. EM, eigengene of the module; GSEA, gene set enrichment analysis; TCGA, The Cancer Genome Atlas.
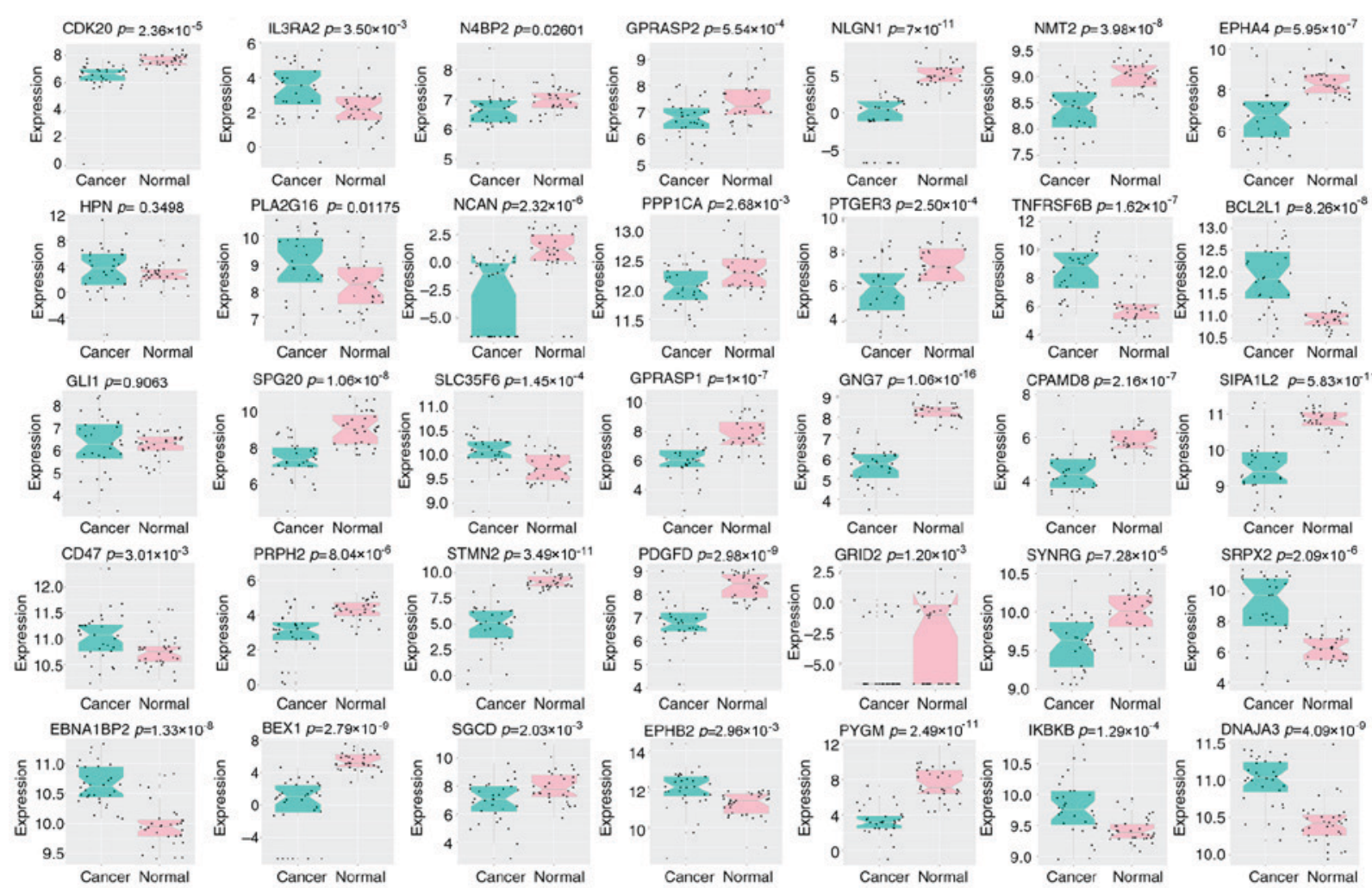

Figure 6. Boxplot of 35 genes significantly affected by carcinogenic dysregulation in TCGA paired data. This illustration indicated that all the genes within this gene signature were significantly dysregulated during colorectal cancer carcinogenesis according to TCGA paired data, with the exception of HPN and GLI1. HPN, hepsin; GLI1, GLI family zinc finger 1; TCGA, The Cancer Genome Atlas.

on Cancer staging information were used to perform Cox analysis, with the exception of GSE28722, since the dataset does not contain sex information. The results indicated that the expression of these 35 genes (as indicated by the EM value) was confirmed as significant in all six cohorts based on the univariate analysis, and as an independent prognostic factor 
A

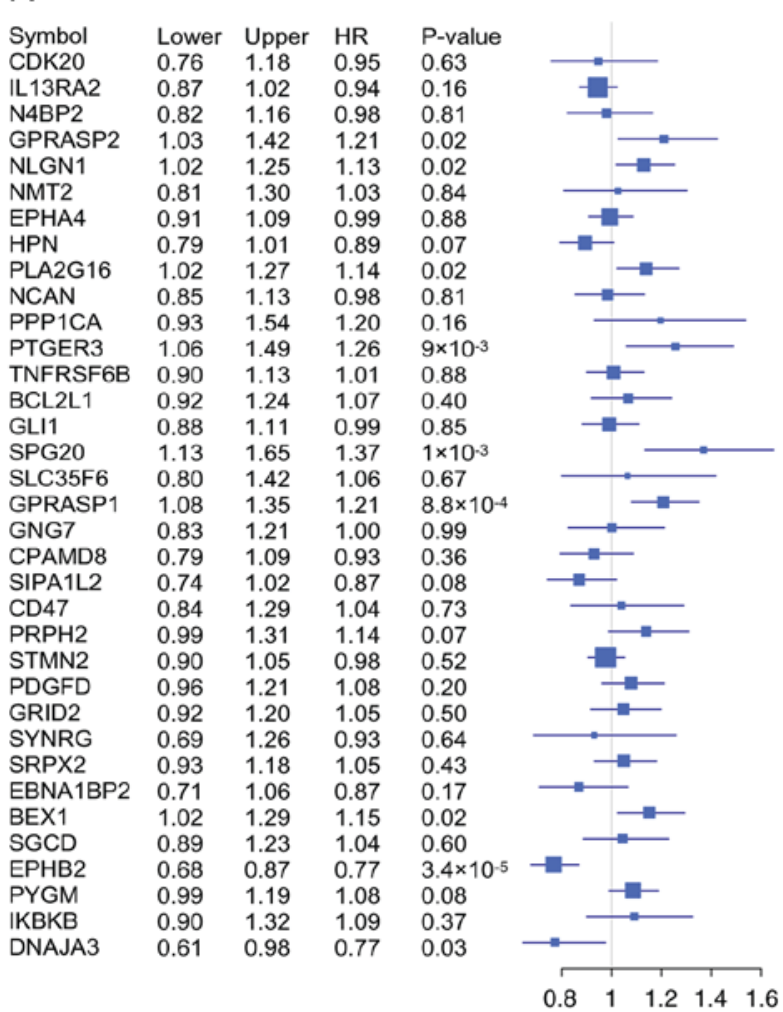

B

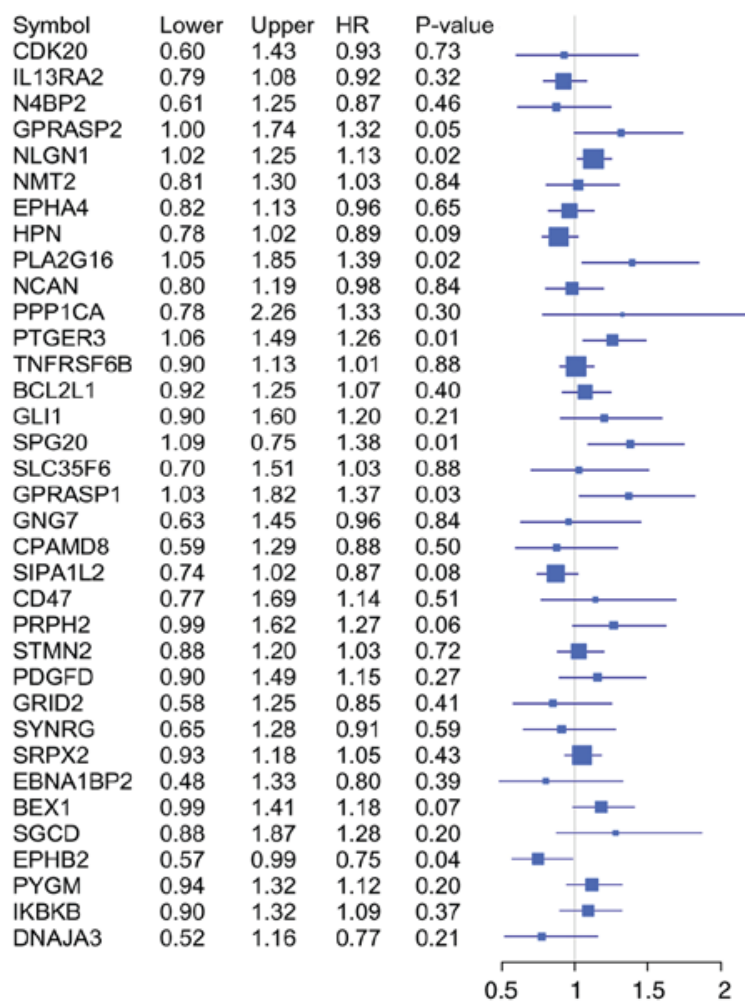

Figure 7. Forest plots of the association between the 35 identified genes and survival in patients with colorectal cancer. (A) Forest plot of the 35 genes with the six independent cohorts using fixed effects model, calculated by pooling all the effect sizes from the six independent cohorts. (B) Forest plot using random effects model. HR, hazard ratio.

in four out of six cohorts, with the exception of GSE71187 $(\mathrm{P}=0.131)$ and $\mathrm{GSE} 17536(\mathrm{P}=0.063)$.

\section{Discussion}

The concept of biological pre-determinism was first proposed in a study by MacDonald, which stated that clinical outcome is possibly pre-determined by the intrinsic natural history of cancer (21). A previous study of a mammary intraepithelial neoplasia model indicated that precancerous cells possess the malignant inclination for latency and distant metastasis, independent of further genetic alterations (22). Invasive behaviors were also discovered in the precancerous stage, suggesting that cancer cell dissemination may happen prior to tumor formation (23). The aforementioned studies indicated that the ability of cancer cells to invade and metastasize may be acquired at a precancerous stage. This concept was also addressed in a previous study (24). Therefore, consistent DEGs continuously activated or inactivated in precancerous and cancer stages may increase the knowledge on the essential molecular dysregulations during carcinogenesis, and aid in the identification of prognostic biomarker and potential therapeutic targets.

GO analysis indicated that consistent DEGs were significantly enriched in immune response and development-associated processes (Fig. 2B). It has been reported that chronic infection and inflammation contribute to $25 \%$ of all cancer types worldwide, particularly in CRC (25). The molecular similarities between malignancies and corresponding developing samples have been reported in respect to transcription factor activity (26), chromatin structural regulation (27) and other signaling pathways (28). Important molecules have been indicated to be activated or inactivated simultaneously in development and carcinogenesis processes. For example, patched 1 is a key regulator for embryonic development and skin carcinogenesis (29). Furthermore, a fetus could also evade maternal immune-surveillance by adopting similar molecular mechanisms to tumors (30). In the present study, GO results of consistent DEGs confirmed that the molecular dysregulations associated with immunity and development regulation may serve an important role in CRC carcinogenesis, as indicated in previous investigations.

In the present study, RWR, a simple and effective computational strategy, was used in a merged biological network to identify genes affected by multistage molecular dysregulation. The RWR algorithm was used to establish gene to disease associations in a priori knowledge-based networks, as it performed superiorly compared with other methods (31-33). The advantage of this computational strategy is subtly combining dysregulation status of one's own data with putatively accepted prior knowledge, rendering genes significantly affected by carcinogenic dysregulation with certain biological and clinical associations. Furthermore, consistent DEGs were weighted with survival association and the edges were weighted with co-expression association. Therefore, genes identified through RWR algorithm possibly contained specific genes undergoing considerable dysregulations during carcinogenesis. Gene signatures derived from one data type should be extensively 
Table I. Univariate and multivariate analyses of survival in patients with colorectal cancer in six test cohorts.

\begin{tabular}{|c|c|c|c|c|}
\hline \multirow[b]{2}{*}{ Factors } & \multicolumn{2}{|c|}{ Univariate cox regression } & \multicolumn{2}{|c|}{ Multivariate cox regression } \\
\hline & $\operatorname{HR}(95 \% \mathrm{CI})$ & P-value & $\operatorname{HR}(95 \% \mathrm{CI})$ & $\mathrm{P}$-value \\
\hline \multicolumn{5}{|l|}{ GSE71187 } \\
\hline Age (years) & $0.959(0.928-0.991)$ & 0.013 & $0.961(0.927-0.997)$ & 0.034 \\
\hline Sex (male/female) & $1.777(0.768-4.115)$ & 0.179 & - & - \\
\hline Stage (III/II) & $2.919(1.073-7.942)$ & 0.036 & $2.261(0.800-6.393)$ & 0.124 \\
\hline $\mathrm{EM}^{\mathrm{a}}$ & $3.068(1.241-7.583)$ & 0.015 & $2.086(0.804-5.411)$ & 0.131 \\
\hline \multicolumn{5}{|l|}{ TCGA } \\
\hline Age (years) & $1.027(1.009-1.045)$ & 0.003 & $1.038(1.019-1.057)$ & $6.090 \times 10^{-5}$ \\
\hline Sex (male/female) & $1.244(0.808-1.915)$ & 0.321 & - & - \\
\hline Stage (III+IV/ I+II) & $2.750(1.759-4.299)$ & $9.190 \times 10^{-6}$ & $3.101(1.949-4.932)$ & $1.769 \times 10^{-6}$ \\
\hline $\mathrm{EM}^{\mathrm{a}}$ & $1.815(1.172-2.809)$ & 0.008 & $1.610(1.031-2.513)$ & 0.036 \\
\hline \multicolumn{5}{|l|}{ GSE14333 } \\
\hline Age (years) & $1.015(1.002-1.028)$ & 0.020 & $1.013(1.001-1.026)$ & 0.042 \\
\hline Sex (male/female) & $0.877(0.651-1.182)$ & 0.390 & - & - \\
\hline Stage $(\text { Duke } C / A+B)^{b}$ & $0.878(0.638-1.207)$ & 0.422 & - & - \\
\hline $\mathrm{EM}^{\mathrm{a}}$ & $1.456(1.072-1.976)$ & 0.016 & $1.395(1.025-1.901)$ & 0.035 \\
\hline \multicolumn{5}{|l|}{ GSE17536 } \\
\hline Age (years) & $1.007(0.989-1.025)$ & 0.473 & - & - \\
\hline Sex (male/female) & $1.105(0.694-1.759)$ & 0.674 & - & - \\
\hline Stage $(\mathrm{III}+\mathrm{IV} / \mathrm{I}+\mathrm{II})$ & $4.220(2.387-7.459)$ & $7.275 \times 10^{-7}$ & $3.934(2.215-6.985)$ & $2.934 \times 10^{-6}$ \\
\hline $\mathrm{EM}^{\mathrm{a}}$ & $1.899(1.180-3.055)$ & 0.008 & $1.576(0.976-2.545)$ & 0.063 \\
\hline \multicolumn{5}{|l|}{ GSE39582 } \\
\hline Age (years) & $1.024(1.012-1.036)$ & $8.793 \times 10^{-5}$ & $1.026(1.014-1.038)$ & $2.356 \times 10^{-5}$ \\
\hline Sex (male/female) & $1.314(0.983-1.756)$ & 0.065 & - & - \\
\hline Stage (III+IV/I+II) & $1.761(1.322-2.347)$ & $1.109 \times 10^{-4}$ & $1.762(1.319-2.354)$ & $1.257 \times 10^{-4}$ \\
\hline $\mathrm{EM}^{\mathrm{a}}$ & $1.457(1.094-1.940)$ & 0.010 & $1.385(1.038-1.847)$ & 0.027 \\
\hline \multicolumn{5}{|l|}{ GSE28722 } \\
\hline Age (years) & $1.017(0.997-1.038)$ & 0.103 & - & - \\
\hline Stage (III+IV/I+II) & $1.205(0.791-1.834)$ & 0.385 & - & - \\
\hline $\mathrm{EM}^{\mathrm{a}}$ & $1.647(1.011-2.688)$ & 0.045 & $1.647(1.011-2.688)$ & 0.045 \\
\hline
\end{tabular}

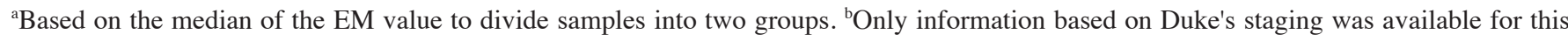
dataset. HR, hazard ratio; CI, confidence interval; TCGA, The Cancer Genome Atlas; EM, eigengene of the module.

tested in other published datasets in order to increase the validity of the whole analysis. Therefore, additional published CRC data were also used to test the validity of these 35 genes significantly affected by carcinogenic dysregulation. A total of six independent cohorts were used to evaluate the prognostic value of these $35 \mathrm{mRNAs}$ significantly affected by carcinogenic dysregulation. Kaplan-Meier survival analysis was conducted to distinguish the actual survival association between the two EM-assigned groups in each dataset. The first principal component captured the greatest amount of total variance in the profiles and was calculated for each patient. Patients were then divided into two groups of equal size based on the rank order of PC1 across their tumor profiles. This method has been frequently used in previous studies (19,34-36). The result indicated that the 35 genes significantly affected by carcinogenic dysregulation performed well in all six independent cohorts (Fig. 5), suggesting a profound prognostic value of the 35 identified genes. The significance of these identified genes in CRC was also supported by a review of the literature. For example, EPH receptor B2 could substantially reduce migration and invasiveness of colonic tumor implants (37), functioning as a key regulator in CRC carcinogenesis and prognostic prediction $(38,39)$. The promoter of spartin, variously methylated in colorectal carcinomas, adenomas and normal mucosa samples (40), was found to be a highly specific and sensitive biomarker for screening CRC (41). Furthermore, Cox analysis revealed that the 35 identified genes were unanimously significant in univariate analysis, validating their association with survival in patients with CRC.

In conclusion, the global expression profiles of human healthy colorectal mucosae, precancerous and CRC samples were downloaded to identify genes with profound prognostic 
information and potential clinical application. Consistent DEGs that continuously differentiated at precancerous and cancer stages were identified. Upregulated consistent DEGs were associated with 'immune response', while downregulated DEGs were mainly associated with 'cell development'. Furthermore, 35 genes significantly affected by carcinogenic dysregulation were successfully identified through a network-based RWR algorithm, and the expression of these genes was significantly associated with CRC patients' survival.

\section{Acknowledgements}

Not applicable.

\section{Funding}

The present study was funded by grants from the Natural Science Foundation of Shandong Province of China (grant no. ZR201702170463).

\section{Availability of data and materials}

The datasets used or analyzed during the current study are available from the corresponding author on reasonable request.

\section{Authors' contributions}

NA, CZ, ZY and XY participated in the study design and the writing of the paper. NA and XY performed the sample selection, algorithm construction and data analysis. All authors have read and approved the manuscript and its contents, and are aware of responsibilities connected to authorship.

\section{Ethics approval and consent to participate}

Not applicable.

\section{Patient consent for publication}

Not applicable.

\section{Competing interests}

The authors declare that they have no conflicts of interest.

\section{References}

1. Brenner H, Kloor M and Pox CP: Colorectal cancer. Lancet 383 : 1490-1502, 2014

2. Weiser MR: AJCC 8th edition: Colorectal cancer. Ann Surg Oncol 25: 1454-1455, 2018.

3. Gonzalez-Pons $\mathrm{M}$ and Cruz-Correa M: Colorectal cancer biomarkers: Where are we now? Biomed Res Int 2015: 149014 , 2015.

4. Schadt EE, Lamb J, Yang X, Zhu J, Edwards S, Guhathakurta D, Sieberts SK, Monks S, Reitman M, Zhang C, et al: An integrative genomics approach to infer causal associations between gene expression and disease. Nat Genet 37: 710-717, 2005.

5. Zhang B, Gaiteri C, Bodea LG, Wang Z, McElwee J, Podtelezhnikov AA, Zhang C, Xie T, Tran L, Dobrin R, et al: Integrated systems approach identifies genetic nodes and networks in late-onset Alzheimer's disease. Cell 153: 707-720, 2013.
6. Chen L, Chu C, Kong X, Huang G, Huang T and Cai YD: A hybrid computational method for the discovery of novel reproduction-related genes. PLoS One 10: e0117090, 2015.

7. Tomczak K, Czerwińska P and Wiznerowicz M: The cancer genome atlas (TCGA): An immeasurable source of knowledge. Contemp Oncol (Pozn) 19: A68-A77, 2015.

8. Chen R, Khatri P, Mazur PK, Polin M, Zheng Y, Vaka D, Hoang CD, Shrager J, Xu Y, Vicent S, et al: A meta-analysis of lung cancer gene expression identifies PTK7 as a survival gene in lung adenocarcinoma. Cancer Res 74: 2892-2902, 2014.

9. Goonesekere NC, Wang X, Ludwig L and Guda C: A meta analysis of pancreatic microarray datasets yields new targets as cancer genes and biomarkers. PLoS One 9: e93046, 2014.

10. An N, Shi X, Zhang Y, Lv N, Feng L, Di X, Han N, Wang G, Cheng $S$ and Zhang K: Discovery of a novel immune gene signature with profound prognostic value in colorectal cancer: A model of cooperativity disorientation created in the process from development to cancer. PLoS One 10: e0137171, 2015.

11. Stolte M: The new Vienna classification of epithelial neoplasia of the gastrointestinal tract: Advantages and disadvantages. Virchows Arch 442: 99-106, 2003.

12. Jorissen RN, Gibbs P, Christie M, Prakash S, Lipton L, Desai J, Kerr D, Aaltonen LA, Arango D, Kruhøffer M, et al: Metastasis-associated gene expression changes predict poor outcomes in patients with dukes stage $\mathrm{B}$ and $\mathrm{C}$ colorectal cancer. Clin Cancer Res 15: 7642-7651, 2009.

13. Smith JJ, Deane NG, Wu F, Merchant NB, Zhang B, Jiang A, Lu P, Johnson JC, Schmidt C, Bailey CE, et al: Experimentally derived metastasis gene expression profile predicts recurrence and death in patients with colon cancer. Gastroenterology 138: 958-968, 2010.

14. Marisa L, de Reyniès A, Duval A, Selves J, Gaub MP, Vescovo L, Etienne-Grimaldi MC, Schiappa R, Guenot D, Ayadi M, et al: Gene expression classification of colon cancer into molecular subtypes: Characterization, validation, and prognostic value. PLoS Med 10: e1001453, 2013.

15. Loboda A, Nebozhyn MV, Watters JW, Buser CA, Shaw PM, Huang PS, Van't Veer L, Tollenaar RA, Jackson DB, Agrawal D, et al: EMT is the dominant program in human colon cancer. BMC Med Genomics 4: 9, 2011.

16. Tusher VG, Tibshirani R and Chu G: Significance analysis of microarrays applied to the ionizing radiation response. Proc Natl Acad Sci USA 98: 5116-5121, 2001.

17. Li J, Roebuck P, Grünewald S and Liang H: SurvNet: A web server for identifying network-based biomarkers that most correlate with patient survival data. Nucleic Acids Res 40: W123-W126, 2012.

18. Ideker T, Ozier O, Schwikowski B and Siegel AF: Discovering regulatory and signalling circuits in molecular interaction networks. Bioinformatics 18 (Suppl 1): S233-S240, 2002.

19. Liu HY, Kho AT, Kohane IS and Sun Y: Predicting survival within the lung cancer histopathological hierarchy using a multi-scale genomic model of development. PLoS Med 3: e232, 2006.

20. Amaral LA, Scala A, Barthelemy M and Stanley HE: Classes of small-world networks. Proc Natl Acad Sci USA 97: 11149-11152, 2000.

21. Macdonald I: Biological predeterminism in human cancer. Surg Gynecol Obstet 92: 443-452, 1951

22. Damonte P, Hodgson JG, Chen JQ, Young LJ, Cardiff RD and Borowsky AD: Mammary carcinoma behavior is programmed in the precancer stem cell. Breast Cancer Res 10: R50, 2008.

23. Rhim AD, Mirek ET, Aiello NM, Maitra A, Bailey JM, McAllister F, Reichert M, Beatty GL, Rustgi AK, Vonderheide RH, et al: EMT and dissemination precede pancreatic tumor formation. Cell 148: 349-361, 2012.

24. Shi X, Zhang Y, Cao B, Lu N, Feng L, Di X, Han N, Luo C, Wang G, Cheng S and Zhang K: Genes involved in the transition from normal epithelium to intraepithelial neoplasia are associated with colorectal cancer patient survival. Biochem Biophys Res Commun 435: 282-288, 2013.

25. Hussain SP and Harris CC: Inflammation and cancer: An ancient link with novel potentials. Int J Cancer 121: 2373-2380, 2007.

26. Hartwell KA, Muir B, Reinhardt F, Carpenter AE, Sgroi DC and Weinberg RA: The Spemann organizer gene, Goosecoid, promotes tumor metastasis. Proc Natl Acad Sci USA 103: 18969-18974, 2006.

27. Sparmann A and van Lohuizen M: Polycomb silencers control cell fate, development and cancer. Nat Rev Cancer 6: 846-856, 2006. 
28. Liu S, Dontu G, Mantle ID, Patel S, Ahn NS, Jackson KW, Suri $\mathrm{P}$ and Wicha MS: Hedgehog signaling and Bmi-1 regulate self-renewal of normal and malignant human mammary stem cells. Cancer Res 66: 6063-6071, 2006.

29. Kang HC, Wakabayashi Y, Jen KY, Mao JH, Zoumpourlis V, Del Rosario R and Balmain A: Ptch1 overexpression drives skin carcinogenesis and developmental defects in K14Ptch(FVB) mice. J Invest Dermatol 133: 1311-1320, 2013.

30. Ridolfi L, Petrini M, Fiammenghi L, Riccobon A and Ridolfi R: Human embryo immune escape mechanisms rediscovered by the tumor. Immunobiology 214: 61-76, 2009.

31. Navlakha $S$ and Kingsford C: The power of protein interaction networks for associating genes with diseases. Bioinformatics 26 1057-1063, 2010

32. Wang XJ, Gulbahce N and Yu HY: Network-based methods for human disease gene prediction. Brief Funct Genomics 10 : 280-293, 2011

33. Zhang C, Li C, Li J, Han J, Shang D, Zhang Y, Zhang W, Yao Q, Han L, Xu Y, et al: Identification of miRNA-mediated core gene module for glioma patient prediction by integrating high-throughput miRNA, mRNA expression and pathway structure. PLoS One 9: e96908, 2014.

34. Chen DT, Hsu YL, Fulp WJ, Coppola D, Haura EB, Yeatman TJ and Cress WD: Prognostic and predictive value of a malignancy-risk gene signature in early-stage non-small cell lung cancer. J Natl Cancer Inst 103: 1859-1870, 2011.
35. Marchion DC, Cottrill HM, Xiong Y, Chen N, Bicaku E, Fulp WJ, Bansal N, Chon HS, Stickles XB, Kamath SG, et al: BAD phosphorylation determines ovarian cancer chemosensitivity and patient survival. Clin Cancer Res 17: 6356-6366, 2011. 36. Hopewell EL, Zhao WP, Fulp WJ, Bronk CC, Lopez AS, Massengill M, Antonia S, Celis E, Haura EB, Enkemann SA, et al: Lung tumor $\mathrm{NF}-\mathrm{\kappa B}$ signaling promotes $\mathrm{T}$ cell-mediated immune surveillance. J Clin Invest 123: 2509-2522, 2013.

37. Senior PV, Zhang BX and Chan ST: Loss of cell-surface receptor EphB2 is important for the growth, migration, and invasiveness of a colon cancer cell line. Int J. Colorectal Dis 25: 687-694, 2010.

38. Drucker A, Arnason T, Yan SR, Aljawad M, Thompson K and Huang WY: Ephrin b2 receptor and microsatellite status in lymph node-positive colon cancer survival. Transl Oncol 6: 520-527, 2013

39. Chen HJ, Wei Z, Sun J, Bhattacharya A, Savage DJ, Serda R, Mackeyev Y, Curley SA, Bu P, Wang L, et al: A recellularized human colon model identifies cancer driver genes. Nat Biotechnol 34: 845-851, 2016.

40. Lind GE, Raiborg C, Danielsen SA, Rognum TO, Thiis-Evensen E, Hoff G, Nesbakken A, Stenmark H and Lothe RA: SPG20, a novel biomarker for early detection of colorectal cancer, encodes a regulator of cytokinesis. Oncogene 30: 3967-3978, 2011.

41. Zhang H, Song YC and Dang CX: Detection of hypermethylated spastic paraplegia-20 in stool samples of patients with colorectal cancer. Int J Med Sci 10: 230-234, 2013. 